\title{
Curación de contenidos en periodismo. Indicadores y buenas prácticas
}

\author{
Javier Guallar*, Rafael Pedraza-Jiménez**, Mario Pérez-Montoro*, Laura Anton**** \\ *Departamento de Biblioteconomía, Documentación y Comunicación Audiovisual de la Universidad de Barcelona. Centro de \\ Investigación en Información, Comunicación y Cultura (CRICC). \\ Correo-e: jguallar@ub.edu ORCID iD: https://orcid.org/0000-0002-8601-3990 \\ Correo-e: perez-montoro@ub.edu ORCID iD: http://orcid.org/0000-0003-2426-8119 \\ **Departamento de Comunicación de la Universidad Pompeu Fabra. Grupo de investigación en Documentación Digital y Co- \\ municación Interactiva (DigiDoc). \\ Correo-e: rafael.pedraza@upf.edu ORCID iD: https://orcid.org/0000-0002-6918-6910 \\ ****Barcelona Televisió (Betevé) \\ Correo-e: lauraansa@gmail.com ORCID iD: https://orcid.org/0000-0001-8615-5932
}

Recibido: 15-12-19; 2a versión: 18-05-20; Aceptado: 23-05-20; Publicado: 27-04-2021

Cómo citar este artículo/Citation: Guallar, J.; Pedraza-Jiménez, R.; Pérez-Montoro, M.; Anton, L. (2021) Curación de contenidos en periodismo. Indicadores y buenas prácticas. Revista Española de Documentación Científica, 44 (2), e296. https://doi. org/ 10.3989/redc.2021.2.1742

Resumen: El artículo presenta un método o sistema para el análisis y la evaluación de la curación de contenidos en medios de comunicación digitales. Este sistema se basa en dos dimensiones (Curación y Contenido) que agrupan una serie de parámetros e indicadores a los cuales se les ha asignado una puntuación. Para cada parámetro se señalan los siguientes elementos: definición, explicación, procedimiento y ejemplos. El elemento "procedimiento" se defiende como una metodología para valorar el uso de la curación de contenidos en un sitio web periodístico, lo que incluye una relación de indicadores, y para cada uno de ellos una pregunta de análisis y una puntuación. El elemento "ejemplos" presenta un conjunto de buenas prácticas de curación de contenidos por parte de medios de comunicación digitales.

Palabras clave: curación de contenidos; curación periodística, curación de noticias; documentación periodística; periodismo digital; fuentes de información; sistemas de análisis; evaluación; indicadores.

\section{Content curation in journalism. Indicators and good practices}

Abstract. This article presents a method or system for the analysis and evaluation of content curation in digital media. The system is based on two dimensions (Content and Curation) that group a series of parameters and indicators to which a score has been assigned. The following elements are indicated for each parameter: definition, explanation, procedure and examples. The "procedure" element is defended as a methodology to assess the use of content curation on a journalistic website, which includes a list of indicators, and an analysis question and a score for each of them. The "examples" element presents a set of good practices for content curation in digital media.

Keywords: content curation; journalistic curation; news curation; news librarianship; digital journalism; information sources; analyses systems; evaluation; indicators.

Copyright: () 2021 CSIC. Este es un artículo de acceso abierto distribuido bajo los términos de la licencia de uso y distribución Creative Commons Reconocimiento 4.0 Internacional (CC BY 4.0). 


\section{INTRODUCCIÓN}

La curación de contenidos en periodismo se puede definir como: "Un complejo de actividades que incluye: 1) búsqueda y monitorización, 2) selección, 3) análisis y verificación, 4) gestión y edición, y 5) caracterización o sense making de informaciones publicadas en la web, con el objetivo de producir o mejorar productos periodísticos, lo que implica 6) la difusión de tales productos a través de plataformas digitales como sitios web de medios de comunicación, blogs y otros medios sociales" (Guallar y Codina, 2018). Las actividades anteriores guardan relación con algunas de las funciones que se han considerado tradicionalmente como propias de la documentación, si bien en el contexto actual del periodismo del siglo XXI, se encuadran cada vez más bajo la denominación de curación de contenidos, como señalan los autores anteriores.

A su vez, se debe señalar que el término "curación de contenidos", o "curaduría de contenidos", es de aparición reciente, situándose su origen en 2009 en el artículo del profesor de marketing Rohit Bhargava "Manifesto for the content curator" (Bhargava, 2009). En dicho trabajo, el autor defendía, en una época caracterizada por el crecimiento exponencial de los contenidos digitales, la necesidad de una actividad profesional especializada en seleccionar el contenido más relevante para una audiencia determinada y en ofrecerlo de la manera más adecuada posible a esa audiencia, aportando un valor añadido.

Desde su aparición en el sector del marketing digital, ese concepto se expande hacia otros campos que tratan con información digital y de manera más destacada, a la biblioteconomía y documentación, el periodismo, la comunicación corporativa, la educación, y la ingeniería y las ciencias informáticas. Diez años después de su aparición, el concepto de curación de contenidos está plenamente presente en la literatura profesional y académica de diversas disciplinas y especialidades. Sin ánimo de exhaustividad, se pueden destacar en la bibliografía especializada, desde trabajos que abarcan la curación en un sentido amplio y transversal, como por ejemplo monografías con un enfoque profesional (Rosenbaum, 2011; Guallar y Leiva-Aguilera, 2013) o ensayístico (Bhaskar, 2016), a trabajos que abordan la aplicación de la curación de contenidos en diferentes sectores, como por ejemplo en bibliotecas (Parra-Valero, 2017; Martínez-Cañadas, 2017). En el ámbito concreto que nos ocupa en esta investigación, la curación de contenidos en periodismo, se pueden destacar entre otros, estudios como los de Bradshaw (2013), Bruns (2018),
Cappelletti Júnior y Domínguez Quintas (2014), Codina (2018), Cui y Liu (2017), Díaz Arias (2015), Guerrini (2013), Guallar (2017 a,b), Guallar y Codina (2018).

El enfoque diferencial de esta investigación respecto a otros trabajos sobre el tema es el de proponer un nuevo sistema para el análisis y la evaluación de la curación de contenidos en productos periodísticos, que se pueda aplicar y sea de utilidad en diversos contextos o escenarios, tanto a nivel académico como profesional.

\section{OBJETIVO, MÉTODO Y PROCEDIMIENTO}

\subsection{Objetivo y justificación}

El objetivo de este artículo es presentar un sistema de análisis basado en un conjunto de parámetros e indicadores que pueda ser útil para la evaluación de la calidad de los productos periodísticos basados en curación de contenidos.

Se justifica su necesidad porque no existe en la bibliografía especializada una propuesta como la que aquí se presenta para la evaluación y el análisis de la curación de contenidos periodísticos, ya que la mayoría de trabajos sobre la temática son descriptivos, teóricos o estudios de caso sobre medios o productos. Excepciones a ello, y como aproximaciones más cercanas a este trabajo, son la propuesta de taxonomía de Guallar (2017a) y la investigación sobre la curación en tres diarios norteamericanos utilizando tres indicadores de Cui y Liu (2017).

En este sentido, este artículo pretende ser una aportación original a la investigación sobre curación de contenidos, que pueda ser de utilidad en diversos escenarios (Rodríguez-Martínez y otros, 2012), como por ejemplo: 1) evaluación de la calidad de la curación de contenidos en sitios web periodísticos, es decir, que permita apreciar de qué manera se ha implementado la curación, con sus puntos fuertes o débiles; 2) análisis académicos de medios de comunicación, que permita establecer rankings, crear pautas o detectar tendencias; 3) ámbito profesional: que permita crear, mejorar o rediseñar productos o servicios de curación de contenidos a partir de los indicadores propuestos.

\subsection{Metodología de investigación}

Para conseguir los fines señalados, en primer lugar, se realizó una extensa revisión bibliográfica tanto sobre curación de contenidos en periodismo como sobre métodos de análisis y evaluación de sistemas y productos de información, en la que se constató la inexistencia de sistemas de 
análisis sobre curación periodística con indicadores de evaluación. En segundo lugar, y a partir de dicha constatación, se decidió utilizar el análisis de sistemas y productos de información (Glaser y Strauss, 1967; Creswell, 2009; Ferran-Ferrer y otros, 2017; Guallar y otros, 2017) y la observación experta (Denzin y Lincoln, 2011) para la identificación y el análisis de una serie de elementos que permitan caracterizar, analizar y evaluar el objeto de estudio. A partir de este análisis, se ha concretado el nuevo sistema para la evaluación de curación de contenidos en medios periodísticos que presenta y describe este trabajo, el sistema CAS (Curation Analysis System).

La investigación sobre análisis de productos y servicios de información cuenta con varios precedentes, aplicados en diversos contextos y temáticas, como por ejemplo: a) sitios web en general (Codina, 2000; Pedraza-Jiménez y otros, 2016; Sanabre y otros, 2018); b) portales de archivos (Perpinyà-Morera y Cid-Leal, 2018); c) sitios web de comercio electrónico (Roig y Pedraza-Jiménez, 2016); d) cibermedios (Rodríguez-Martínez y otros, 2010, 2012); e) hemerotecas de prensa (Guallar y Abadal, 2009; Guallar y otros, 2012); f) bases de datos de prensa (Linares y otros, 2016); g) imágenes para prensa (Carbonell y Pedraza-Jiménez, 2016); h) webs de televisiones (Díaz Campo, 2014); i) archivos de televisiones en internet (Anton y Guallar, 2014); j) software de archivos audiovisuales (Cascón-Katchadourian y otros, 2018).

Nuestra propuesta metodológica ha tenido en cuenta todas las aportaciones señaladas, y de ellas ha prestado una atención especial a aquellos sistemas de análisis y evaluación basados en parámetros e indicadores, como el que se expone en detalle en Pedraza-Jiménez y otros (2016). En un sistema de análisis y evaluación de este tipo, los parámetros hacen referencia a los aspectos generales de los productos periodísticos susceptibles de estudio. Cada parámetro consta de un conjunto de indicadores, que son los aspectos concretos cuyo análisis nos permitirá emitir un juicio de valor sobre el parámetro en el que se integran.

En una metodología de este tipo, otra cuestión relevante a considerar es el sistema de puntuación que se asigna a los indicadores. Puede ser binario (0-1), cuando se evalúa la presencia o ausencia de una característica, o de puntuación múltiple (0-3), cuando se desea realizar una evaluación exhaustiva de las características estudiadas, valorándolas: mal (0); regular (1); bien (2); o muy bien (3). En nuestra propuesta se combinan ambas opciones, como se detallará más adelante.
Para obtener los indicadores que se presentarán a continuación se desarrollaron dos tareas. Por un lado, se realizó una observación de los productos de curación de contenidos en los principales medios digitales españoles. Para la selección de medios se consultaron los rankings de audiencia de la prensa digital española de ComScore (DIR Confidencial, 2019) y de OJD Interactiva (OJD Interactiva 2019), obteniendo una lista de siete medios tradicionales o legacy media (ABC, El Mundo, El País, El Periódico, La Razón, La Vanguardia y 20 Minutos) y siete nativos digitales (El Confidencial, El Diario.es, El Español, El Nacional, Nació Digital, OK Diario y Público). La elección de esta muestra de análisis se hizo con la intención de realizar la observación en un conjunto importante, y a la vez acotado, de medios periodísticos. En cuanto a la identificación de parámetros e indicadores de calidad, se realizó a partir de un análisis en profundidad de las características de las noticias de estos diarios, lo que permitió la identificación de 2 dimensiones de análisis (curación y contenido), 9 parámetros (o aspectos generales de evaluación), y 33 indicadores (que permiten valorar la calidad de los parámetros identificados). La monitorización de dichos cibermedios se realizó entre marzo y octubre de 2019.

Por otra parte, se analizaron en profundidad las propuestas existentes en la literatura especializada y que, con diferentes enfoques, han empleado diversos elementos para analizar el uso de la curación de contenidos. En concreto los trabajos que se han estudiado como principales referentes son los de Deshpande (2013), Barnhurst (2013), Cui y Liu (2017), Guallar (2017a), Martínez-Cañadas (2017), Orero y Cebrián-Enrique (2019). A partir de esos trabajos, se han adaptado, modificado y refinado algunos de los indicadores que ahí se utilizan, y se han propuesto otros nuevos, agrupándolos en nuestro sistema. En el apartado siguiente en el que se presenta el sistema de análisis se detallan, para cada parámetro, las referencias bibliográficas consideradas y las aportaciones realizadas.

Por último, con respecto a la bibliografía señalada acerca de métodos de análisis y evaluación de productos y servicios de información, el presente artículo se puede situar en línea, en cuanto a tipo de propuesta y de estructura, con trabajos anteriores, como los de Guallar y Abadal (2009), Rodríguez-Martínez y otros (2012), o Linares y otros (2016). Como en los casos citados, se presentará a continuación un sistema de análisis con ejemplos de buenas prácticas. 


\section{SISTEMA DE ANÁLISIS CAS Y EJEMPLOS DE BUENAS PRÁCTICAS}

El sistema de análisis que presentamos, al que hemos denominado CAS (Curation Analysis System), se basa en parámetros e indicadores, que se han agrupado a su vez en las dimensiones Contenido y Curación, tal y como muestra la tabla 1.

Tabla I. Sistema CAS (Curation Analysis System). Tabla resumen de dimensiones, parámetros e indicadores

\begin{tabular}{|c|c|}
\hline \multicolumn{2}{|l|}{ A. Contenido } \\
\hline $\begin{array}{l}\text { A1. Número de } \\
\text { contenidos }\end{array}$ & A1.1. Cantidad \\
\hline A2. Rango temporal & $\begin{array}{l}\text { A2.1. Información retrospectiva } \\
\text { o atemporal } \\
\text { A2.2. Información reciente } \\
\text { A2.3. Información actual } \\
\text { A2.4. Información en tiempo real }\end{array}$ \\
\hline A3. Procedencia & $\begin{array}{l}\text { A3.1. Contenido propio } \\
\text { A3.2. Contenido externo }\end{array}$ \\
\hline $\begin{array}{l}\text { A4. Fuente según } \\
\text { tipo de } \\
\text { organización }\end{array}$ & $\begin{array}{l}\text { A4.1 Fuentes oficiales } \\
\text { A4.2 Fuentes corporativas } \\
\text { A4.3 Medios de comunicación } \\
\text { A4.4 Ciudadanos } \\
\text { A4.5 Comunidades online }\end{array}$ \\
\hline $\begin{array}{l}\text { A5. Fuente según } \\
\text { morfología }\end{array}$ & $\begin{array}{l}\text { A5.1 Sitios web } \\
\text { A5.2 Blogs } \\
\text { A5.3 Redes sociales } \\
\text { A5.4 Fuentes secundarias }\end{array}$ \\
\hline \multicolumn{2}{|l|}{ B. Curación } \\
\hline B1. Autoría & B1.1. Autoría \\
\hline $\begin{array}{l}\text { B2. Técnica de } \\
\text { caracterización }\end{array}$ & $\begin{array}{l}\text { B2.1. Retitular } \\
\text { B2.2. Resumir } \\
\text { B2.3. Comentar } \\
\text { B2.4. Citar } \\
\text { B2.5. Storyboarding } \\
\text { B2.6. Paralelizar }\end{array}$ \\
\hline B3. Integración & $\begin{array}{l}\text { B3.1. Hipervínculo en el texto } \\
\text { B3.2. Hipervínculo en listas o } \\
\text { etiquetas } \\
\text { B3.3. Contenido incrustado }\end{array}$ \\
\hline $\begin{array}{l}\text { B4. Función } \\
\text { periodística }\end{array}$ & $\begin{array}{l}\text { B4.1. Sin modificar } \\
\text { B4.2. Describir } \\
\text { B4.3. Contextualizar } \\
\text { B4.4. Interpretar } \\
\text { B4.5. Citar fuente } \\
\text { B4.6. Citar autor } \\
\text { B4.7. Llamar a la acción }\end{array}$ \\
\hline
\end{tabular}

A continuación, se detallará el sistema. A tal fin, para cada parámetro se facilitan las siguientes informaciones:

- Definición: breve definición del parámetro

- Explicación: descripción detallada, así como indicaciones y discusión acerca de su origen o presencia en la literatura.

- Procedimiento: metodología a seguir para valorar su uso en un sitio web periodístico, que incluye la relación de indicadores, y para cada uno de ellos la pregunta de análisis y la puntuación.

- Ejemplos: se muestran ejemplos reales de buenas prácticas por parte de medios de comunicación digitales

Por último, conviene hacer una precisión en cuanto al uso de este sistema. Este nuevo sistema de análisis pretende ser exhaustivo, es decir, pretende cubrir todos los aspectos que afectan a la calidad de la curación en un medio periodístico y en él se propone un método de puntuación que no prioriza ninguno de los aspectos de calidad identificados. No obstante, quedará a criterio del analista o evaluador que lo emplee, su utilización total o parcial (si sólo está interesado en una parte de los parámetros identificados), así como la modificación de las puntuaciones propuestas si desea dar más importancia a unos indicadores que a otros. Por ejemplo, en el parámetro de Rango temporal, que hace referencia, como se verá, al marco temporal de los contenidos curados; un evaluador podría dar más importancia al contenido retrospectivo que al contenido en tiempo real y puntuarlos de manera diferente, al considerar que solo los mejores medios utilizan sistemas de información retrospectiva.

\section{A. Dimensión Contenido}

\section{A1. Parámetro: Número de contenidos cu-} rados

\section{Definición:}

Cantidad de contenidos curados existentes en un producto periodístico.

\section{Explicación:}

En este trabajo denominamos productos periodísticos a aquellas unidades de análisis que se pretendan estudiar con esta metodología en los medios de comunicación; por ejemplo, pueden ser las noticias de la página de inicio, las noticias de una determinada sección, las newsletters, etc. Este parámetro se debe aplicar siempre en el contexto de comparar productos homogéneos entre sí; por ejemplo, si queremos analizar las newsletters de varios medios, las noticias de la página de inicio de varios medios, etc. Para identificar cuáles son los contenidos curados dentro de un determinado producto periodístico, se debe tener en cuenta si existe un enlace, ya sea en el texto, en una imagen o en forma de contenido incrustado, hacia un contenido que es independiente y externo al que se está analizando. Si no existe ese enlace que da acceso a otro contenido original, aun cuando se le mencione, no se puede considerar que existe contenido curado. 
Una de las distinciones más claras que se pueden establecer entre unos tipos de productos periodísticos de curación de otros es la cantidad de contenidos curados que ofrecen. En este sentido, la cantidad se puede considerar un indicador de calidad. Este parámetro estaba presente en la taxonomía de Guallar (2017a), como distinción entre los productos que curan un solo contenido de los que curan varios, y sin puntuación. En nuestro sistema de evaluación se le asigna una puntuación en varios niveles al considerar la cantidad de contenidos curados disponibles dentro de un mismo producto como un criterio de calidad del mismo.

\section{Procedimiento:}

En este parámetro solo se considera un indicador, del mismo nombre.

\section{- A1.1. Cantidad de contenidos curados.}

Pregunta de análisis: ¿Cuántos contenidos curados tiene el producto analizado?

Puntuación: 0-inexistente; 1-tercil inferior; 2-tercil medio; 3-tercil superior

Se debe poner en primer lugar en contexto el producto analizado con otros del mismo tipo: por ejemplo, la página de inicio de un medio digital, una determinada sección del medio, una newsletter, etc. A partir de ahí se establece una puntuación en tres niveles (tres terciles), considerando el número más alto de fuentes curadas que se pueda tomar como referencia de buenas prácticas como el nivel máximo y dividiéndolo en tres partes: obtienen 3 puntos los productos con un número de contenidos situado en el tercil superior, 2 para el medio, uno para el bajo.

\section{Ejemplos:}

- "Mientras Dormías", newsletter de El Español, 27 junio 2019.

A1: 3 puntos

- "Las 7 y media", newsletter de $A B C, 27$ junio 2019

\section{A1: 1 punto}

Para una newsletter periodística se podría establecer la siguiente propuesta a partir de la observación de la prensa digital: para un rango de 1-10 contenidos curados por newsletter, 1 punto; para 11-20 contenidos 2 puntos; para más de 21 contenidos, 3 puntos.

Aplicando este sistema de puntuación a la newsletter "Mientras dormías" del diario El Español, que el día 27 de junio de 2019 presentaba 30 contenidos curados, se evaluaría asignándole 3 puntos en este indicador. Siguiendo el mismo procedimiento, "Las 7 y media" del diario $A B C$ recibiría 1 punto, ya que en su edición de ese mismo día solo aparecían 8 contenidos curados.

A2. Parámetro: Rango temporal del contenido curado

\section{Definición:}

Rango temporal en el que se enmarca el contenido curado atendiendo a la fecha en la que fue publicado originalmente.

\section{Explicación:}

Se distinguen cuatro rangos temporales, a partir de las investigaciones previas de Guallar, 2017a y b: información retrospectiva o atemporal (de meses o de años anteriores); información reciente (de los últimos días o semanas), información actual (de las últimas horas), e información en tiempo real.

En el caso de la curación periodística es fácil y útil diferenciarla en cada uno de estos rangos temporales: el primero de ellos (información retrospectiva) está muy estrechamente relacionado con la práctica tradicional de la documentación periodística, mientras que el resto ha crecido exponencialmente en los últimos años a medida que los medios sociales y la información en tiempo real han ido ganando protagonismo en los hábitos informativos de las audiencias.

\section{Procedimiento:}

Se consideran cuatro indicadores para cada uno de los rangos temporales establecidos.

\section{- A2.1. Información retrospectiva o atemporal.}

Pregunta de análisis: ¿El producto tiene contenidos curados retrospectivos, de meses o años anteriores, o son contenidos atemporales?

Puntuación: 0-No; 1-Sí

- A2.2. Información reciente.

Pregunta de análisis: ¿El producto tiene contenidos curados recientes, de los últimos días o semanas?

Puntuación: 0-No; 1-Sí

\section{- A2.3. Información actual.}

Pregunta de análisis: ¿El producto tiene contenidos curados actuales, de las últimas horas?

Puntuación: 0-No; 1-Sí

- A2.4. Información en tiempo real.

Pregunta de análisis: ¿El producto tiene contenidos curados en tiempo real? 


\section{Puntuación: 0-No; 1-Sí}

Los indicadores resultantes de la aplicación de este parámetro no son excluyentes, sino compatibles entre sí y será un elemento de calidad de un producto periodístico su capacidad de ofrecer el máximo posible de los mismos. En ese caso, el mínimo de puntuación que puede obtener un producto de curación en este parámetro es 1 y el máximo, 4

\section{Ejemplos:}

- "Planes para el Valle de los Caídos después de Franco" (Natalia Junquera, El País, 12 octubre 2019) <https://elpais.com/politica/2019/10/12/ actualidad/1570901355_289484.html>

\section{A2: 3 puntos}

En esta noticia predomina la curación de contenidos publicados en meses y años anteriores, concretamente hasta 2011, lo que supone valorar con 1 punto la presencia de contenido retrospectivo. Además, se ha curado información de los últimos días, por lo que se valora con 1 punto la presencia de información reciente. Por último, también se ha curado contenido publicado durante las últimas 24 horas, por lo que se valora con 1 punto la presencia de contenido actual. De este modo, este parámetro obtiene un total de 3 puntos que, como acabamos de ver, procede de los indicadores: Información retrospectiva o atemporal, Información reciente, e Información actual.

- "Los independentistas calientan motores con una protesta en Sants" (Guillem Sànchez, El Periódico, 13 octubre 2019) <https://www.elperiodico. com/es/politica/20191013/protesta-independentista-estacion-sants-7679413>

\section{A2: 3 puntos}

Véase en este caso una noticia que se sigue en directo, que incluye la curación de las publicaciones en Twitter del autor de la información y de otros usuarios junto a contenidos publicados en las últimas horas y un contenido atemporal. En este parámetro se obtiene un total de 3 puntos, correspondientes a los indicadores: Información en tiempo real, Información actual, e Información retrospectiva o atemporal.

\section{A3. Parámetro: Procedencia del contenido curado \\ Definición:}

Procedencia del contenido curado desde el punto de vista del medio u organización que lo publica.

\section{Explicación:}

El contenido curado puede tener dos procedencias desde el punto de vista de la organización que produ- ce el producto noticioso: puede ser contenido externo al propio medio, o bien contenido propio, es decir, publicado previamente por el propio medio digital. Tradicionalmente, la prensa digital ha privilegiado los enlaces hacia el contenido propio muy por encima de los enlaces al contenido externo y, si bien algunas investigaciones señalan una ligera corrección (Karlsson y otros, 2015), el contenido curado propio suele ser muy mayoritario en los enlaces de los productos periodísticos (Fondevila-Gascón y Segura-Jiménez, 2012; Orero y Cebrian-Enrique, 2019).

\section{Procedimiento:}

La valoración de este parámetro se realiza en función de dos indicadores, uno para cada una de las categorías establecidas.

\section{- A3.1. Contenido propio.}

Pregunta de análisis: ¿El producto tiene contenidos curados propios?

Puntuación: 0-No; 1-Sí

\section{- A3.2. Contenido externo.}

Pregunta de análisis: ¿El producto tiene contenidos curados externos?

\section{Puntuación: 0-No; 1-Sí}

Los indicadores de este parámetro no son excluyentes, sino compatibles entre sí $y$, considerando el contexto señalado antes, será un elemento de calidad para un producto periodístico ofrecer los dos tipos de contenido. El mínimo de puntuación que se puede obtener en este parámetro es 1 y el máximo, 2 .

\section{Ejemplo:}

"Sánchez asume el peso del 10N: se multiplica en medios y mítines para activar al votante" (Juanma Romero, El Confidencial, 5 octubre 2019)

<https://www.elconfidencial.com/elecciones-generales/2019-10-05/pedro-sanchez-peso-campana-multiplica-medios-mitines-movilizacion_2270116/>

\section{A3: 2 puntos}

El artículo combina los contenidos curados publicados previamente por el propio medio con los de otras cabeceras, hasta un total de 8: de Antena $3 \mathrm{TV}$, La Razón, Aragón TV, PSOE, etc.). De esta forma, ofrece al lector una importante variedad de puntos de vista acerca de la noticia. En el parámetro se obtienen 2 puntos correspondientes a la evaluación positiva de los dos indicadores: contenido propio y externo.

A4. Parámetro: Fuente del contenido curado según tipo de organización 


\section{Definición:}

Tipo de organización de la fuente productora del contenido curado

\section{Explicación:}

Existen diversas propuestas de clasificación para las fuentes de información utilizadas en periodismo, buena parte de ellas realizadas desde la especialidad de la documentación periodística y ahora también, de la curación periodística. A partir del análisis de, entre otros, los trabajos previos de Barnhurst, 2013; Cui y Liu 2017; Guallar y Codina 2018; Orero y Cebrian-Enrique, 2019, se presenta una propuesta que considera dos parámetros diferenciados para las fuentes, según la organización productora y según la estructura de la misma. En este apartado A4 de tipo de organización, se distingue entre fuentes oficiales (de la Administración pública), corporativas (de empresas u otras organizaciones privadas), medios de comunicación, ciudadanos particulares y comunidades online (wikis colaborativos, hashtags, foros online...).

\section{Procedimiento:}

Conforman este parámetro cinco indicadores, uno para cada una de las cinco categorías establecidas.

- A4.1. Contenido procedente de fuentes oficiales.

Pregunta de análisis: ¿El producto tiene contenidos curados procedentes de fuentes oficiales, de las administraciones públicas?

Puntuación: 0-No; 1-Sí

- A4.2. Contenido procedente de fuentes corporativas.

Pregunta de análisis: ¿El producto tiene contenidos curados procedentes de fuentes corporativas, de empresas u otras organizaciones privadas?

Puntuación: 0-No; 1-Sí

- A4.3. Contenido procedente de medios de comunicación.

Pregunta de análisis: ¿El producto tiene contenidos curados procedentes de medios de comunicación?

Puntuación: 0-No; 1-Sí

- A4.4. Contenido procedente de ciudadanos particulares.

Pregunta de análisis: ¿El producto tiene contenidos curados procedentes de fuentes personales, de ciudadanos particulares?

Puntuación: 0-No; 1-Sí
- A4.5. Contenido procedente de comunidades online.

Pregunta de análisis: ¿El producto tiene contenidos curados procedentes de comunidades online (wikis colaborativos, hashtags, foros online...)

\section{Puntuación: 0-No; 1-Sí}

Este parámetro, como en los dos casos anteriores, tiene indicadores no excluyentes y considera la variedad de tipos de fuentes en los contenidos curados como un criterio de calidad. Así, una puntuación de 1 indicará una mínima variedad de tipos de fuentes curadas por parte del producto analizado, mientras que 5 puntos, significaría una gran variedad de tipos de fuentes curadas.

\section{Ejemplos:}

-"¿Starbucks en Westeros? Las bromas sobre la aparición de un vaso en 'Game of Thrones'" (Diana Rodríguez, El País, Verne, 7 mayo 2019) https://verne.elpais.com/verne/2019/05/06/mexico/1557171719_137538.html

\section{A4: 3 puntos}

El contenido curado en esta entrada toma en consideración publicaciones realizadas por usuarios en sus perfiles en redes sociales, junto con contenidos publicados por fuentes corporativas en redes sociales (Starbucks, Game of Thrones), así como noticias publicadas en el medio. En consecuencia, la fuente analizada obtiene en este parámetro 3 puntos, que corresponden a los indicadores: Ciudadanos particulares, Fuentes corporativas y Medios de comunicación.

-"El abogado que convirtió a miles de apátridas en ciudadanos con derechos en Kirguistán, Premio Nansen de ACNUR" (Eldiario.es, 2 octubre 2019)

$<$ https://www.eldiario.es/desalambre/Kirguistan-Azizbek-Ashurov-Premio-ACNUR_0_947706060. html>

\section{A4: 2 puntos}

Esta curación se basa fundamentalmente en las fuentes de información oficiales de ACNUR, combinadas con noticias publicadas en medios. Se obtiene así 2 puntos, correspondientes a los indicadores de Fuentes Oficiales y de Medios de comunicación.

A5. Parámetro: Fuente del contenido curado según su morfología

\section{Definición:}

Este parámetro evalúa la morfología o características de formato de la fuente del contenido curado.

\section{Explicación:}


A partir del análisis de la misma literatura que se ha comentado para el parámetro anterior A4, en este apartado A5 de tipo de organización de la fuente de contenido atendiendo a su morfología, se distingue entre: sitios web; blogs; redes sociales; y fuentes secundarias (como bases de datos, catálogos...).

Es importante señalar que estos dos parámetros A4 y A5 de fuentes del contenido curado según el tipo de organización y según su morfología, no son parámetros repetitivos, pues aunque ambos se refieren a fuentes de información, estas se han clasificado en base a criterios distintos. Así, por ejemplo, el contenido curado procedente de un blog (parámetro A5), podría ser de: una fuente oficial, una fuente corporativa, un medio de comunicación o un ciudadano particular (parámetro A4).

\section{Procedimiento:}

Para la valoración de este parámetro se consideran cuatro indicadores, uno para cada una de las categorías establecidas.

- A5.1. Contenido procedente de sitios web.

Pregunta de análisis: ¿El producto tiene contenidos curados procedentes de sitios web?

Puntuación: 0-No; 1-Sí

- A5.2. Contenido procedente de blogs.

Pregunta de análisis: ¿El producto tiene contenidos curados procedentes de blogs?

Puntuación: 0-No; 1-Sí

- A5.3. Contenido procedente de plataformas sociales.

Pregunta de análisis: ¿El producto tiene contenidos curados procedentes de plataformas sociales?

Puntuación: 0-No; 1-Sí

- A5.4. Contenido procedente de fuentes secundarias.

Pregunta de análisis: ¿El producto tiene contenidos curados procedentes de fuentes secundarias, como bases de datos, catálogos...?

Puntuación: 0-No; 1-Sí

Como en el parámetro anterior, aquí se valora la variedad de tipos de fuentes según su morfología en los contenidos curados como un criterio de calidad, y así, una puntuación total en este parámetro de 1 indicará la mínima variedad de fuentes, y el 4, la máxima variedad posible.

\section{Ejemplos}

- "En los barrios pobres, las frutas y plantas salvan vidas" (Roberto Palomo, El País, 14 noviembre 2019)

\section{A5: 3 puntos}

$<$ https://elpais.com/elpais/2019/11/14/planeta_futuro/1573741258_140453.html>

En esta noticia se enlazan contenidos procedentes de sitios web, de blogs y de bases de datos. Por ello, se obtienen en este parámetro 3 puntos, que se corresponden con los indicadores de: contenido procedente de sitios web, de blogs, y de fuentes secundarias.

- "Polémica en Argentina por una foto de una rider con su bebé" (El Periódico, 6 octubre 2019)

<https://www.elperiodico.com/es/sociedad/20191006/la-polemica-en-argentina-por-unafoto-de-una-rider-con-su-bebe-7669208>

\section{A5: 2 puntos}

En esta curación destacan los contenidos publicados en dos plataformas sociales (Facebook y Twitter) que se complementan con un enlace a un sitio web. En consecuencia, se obtienen 2 puntos en este parámetro, que se refieren a los indicadores de: contenido procedente de plataformas sociales, y de contenido procedente de sitios web.

\section{B. Dimensión curación}

\section{B1. Parámetro: Autoría de la curación Definición :}

Este parámetro analiza si existe una autoría declarada del producto de curación estudiado.

\section{Explicación:}

Un elemento clave y fundamental para la evaluación de la calidad de cualquier producto o servicio digital es la identificación clara e inequívoca de su autoría. Así ha quedado establecido en buena parte de la bibliografía especializada en métodos de análisis o evaluación, ya desde las primeras propuestas existentes, como la de Codina (2000).

\section{Procedimiento:}

En este parámetro se considera un indicador de valoración de la autoría del producto periodístico.

- B1.1. Autoría de la curación.

Pregunta de análisis: ¿Es visible la autoría del producto analizado?

Puntuación: 0-No; 1-Sí 
En el caso de un producto periodístico de curación de contenidos, la valoración de su autoría tiene consideraciones similares a la de cualquier otro contenido digital. Así, se considera un elemento de calidad si está disponible de manera clara y precisa su identificación para el lector.

\section{Ejemplo}

- "Mientras dormías, 31 de julio de 2019" (Paolo Fava, El Español, 31 julio 2019) https://www. elespanol.com/newsletter/

\section{B1: 1 punto}

Esta newsletter diaria de curación de contenidos explicita claramente su autoría, a cargo de Paolo Fava. Ello la distingue y la destaca sobre otros boletines periodísticos que son más bien de tipo automatizado y no tienen una autoría identificada. Por tanto, se obtiene un punto en este parámetro.

B2. Parámetro: Técnicas de caracterización de contenidos o sense making

\section{Definición:}

En este parámetro se valora la utilización visible en el producto curado de técnicas de sense making o caracterización de contenidos.

\section{Explicación:}

En la bibliografía especializada sobre curación de contenidos se enfatiza la importancia de lo que se suele denominar sense making o caracterización de contenido (Deshpande, 2013; Guallar y Leiva-Aguilera, 2013; Martínez-Cañadas, 2017); esto es, se trata de añadir valor al contenido curado que se ofrece a la audiencia, poniéndolo en contexto mediante una o varias de las técnicas descritas en la bibliografía. Deshpande (2013) identifica seis técnicas en una taxonomía que ha tenido una considerable influencia, siendo adaptada y revisada posteriormente. Las seis técnicas de Deshpande, de menor a mayor dificultad para el curador según este autor, son: extractar, retitular, resumir o comentar, citar, storyboarding y paralelizar. La primera de ellas es poco más que una extracción automatizada, por lo que se ha prescindido de la misma en este método de análisis al considerar que no aporta valor más allá de la propia selección del contenido a curar. En esta investigación sí se consideran todas las otras, añadiendo además por nuestra parte una diferenciación entre resumir y comentar:

retitular: (habitualmente, solo para cuando se trata de una única fuente) curar el contenido con un título diferente al original resumir: ofrecer un texto de resumen informativo u objetivo acerca del contenido curado

comentar: presentar un texto de resumen en un tono personal, subjetivo o de opinión

citar: incluir una cita textual del contenido curado

storyboarding: unir en un mismo producto diversas piezas de contenido en formatos diversos (por ejemplo, textos de artículos, fotos, tuits, videos incrustados...) intercalando texto propio.

paralelizar: presentar dos o más contenidos que no parecen tener vinculación entre ellos pero que el curador relaciona, justificando y explicando dicha vinculación.

Estas técnicas pueden presentarse independientemente o combinadas en un mismo producto de curación. Por ejemplo, las dos últimas, que requieren de gran elaboración, normalmente comportan su uso junto con otras técnicas de las anteriores.

\section{Procedimiento:}

Para la evaluación de este parámetro se consideran seis indicadores, uno para cada una de las categorías descritas anteriormente.

\section{-B2.1. Retitular.}

Pregunta de análisis: ¿El producto analizado retitula el título original del contenido curado?

Puntuación: 0-No; 1-Sí

\section{-B2.2. Resumir.}

Pregunta de análisis: ¿El producto analizado contiene un texto de resumen informativo u objetivo del contenido curado?

Puntuación: 0-No; 1-Sí

\section{-B2.3. Comentar.}

Pregunta de análisis: ¿El producto analizado contiene un texto de resumen personal, subjetivo o de opinión acerca del contenido curado?

Puntuación: 0-No; 1-Sí

-B2.4. Citar.

Pregunta de análisis: ¿El producto analizado contiene una cita textual del contenido curado?

Puntuación: 0-No; 1-Sí

-B2.5. Storyboarding.

Pregunta de análisis: ¿El producto analizado une diversas piezas de contenido en formatos diversos?

Puntuación: 0-No; 1-Sí 


\section{- B2.6. Paralelizar.}

Pregunta de análisis: ¿El producto analizado relaciona dos o más contenidos que no tenían antes vinculación entre ellos?

Puntuación: 0-No; 1-Sí

Este parámetro para la evaluación de la calidad de la curación puede hacer variar la puntuación del producto evaluado entre 0 puntos, si la curación es totalmente automatizada, y un máximo de 6 puntos, en el caso de combinar todas las técnicas presentadas. No obstante, una alta combinación de estas técnicas es muy poco frecuente.

\section{Ejemplos:}

- "El Despertador. 30 de mayo de 2019" (Paolo Faova, El Español, 30 mayo 2019)

https://www.elespanol.com/newsletter/

\section{B2: 3 puntos}

En esta newsletter se han aplicado tres técnicas de las descritas anteriormente: se ha realizado un resumen descriptivo de contenidos (Resumir), se ha mostrado la opinión del autor (Comentar) y se han añadido citas textuales (Citar). Se obtienen así 3 puntos en este parámetro, procedentes de los indicadores: Resumir, Comentar y Citar.

- "Cargas en Via Laietana y batalla campal en el aeropuerto de El Prat" (EI Mundo, 14 octubre 2019) <https://www.elmundo.es/cataluna/2019/10/14/ 5da407d321efa0a1248b468a.html>

\section{B2: 3 puntos}

En el seguimiento de un evento de gran interés noticioso es habitual que los medios digitales publiquen noticias que se actualizan constantemente en directo, a medida que el evento evoluciona. En estos casos, la técnica del storyboarding es muy adecuada, ya que integra contenido de diversas fuentes y formatos de plataformas sociales, junto a la narración periodística de lo que va aconteciendo. En el caso del ejemplo referenciado, el formato externo más empleado han sido tweets, sobre todo de entidades y de políticos, así como videos y un gráfico interactivo del propio medio. Junto a ello, la descripción de la evolución de la jornada en un tono informativo encaja con la técnica de resumir y también se incluyen algunas declaraciones textuales. Se obtienen así 3 puntos, procedentes de los indicadores: Storyboarding, Resumir y Citar.

\section{B3. Parámetro: Integración del contenido} curado

\section{Definición:}

Este parámetro valora la forma en que se integra el contenido curado en el producto analizado

\section{Explicación:}

Nos fijamos exclusivamente en la forma del punto de acceso a cada contenido curado y no en la totalidad del producto periodístico de curación que se está analizando (artículo, página, sección, newsletter...). Se ha partido en este caso de la taxonomía de Guallar (2017a), y se ha modificado a partir de la observación de medios digitales realizada para esta investigación. En concreto, se distingue:

Hipervínculo dentro de un texto (el sistema más habitual en periodismo)

Hipervínculo en un apartado o sección de lista de enlaces o lista de etiquetas

Contenido incrustado (embedded): el contenido se integra dentro del producto periodístico y se puede consultar con las características propias de la publicación en la plataforma original (visionar, escuchar, etc.) sin salir del propio producto. Es el sistema habitual para visionar contenidos de algunas plataformas sociales como YouTube, Twitter...

En este parámetro es importante señalar que no se considera curación cuando en un determinado producto periodístico se hacen referencias a informaciones publicadas previamente a las cuales no se ofrece acceso. Es decir, si hay referencias a contenidos pero no existen hipervínculos o contenido incrustado, la puntuación sería 0 . Asimismo, como en otros casos, se pueden presentar varias de estas posibilidades combinadas en un mismo producto de curación.

\section{Procedimiento:}

Para la valoración de este parámetro se utilizan tres indicadores, uno para cada una de las categorías establecidas.

\section{- B3.1. Hipervínculo dentro del texto.}

Pregunta de análisis: ¿EI producto integra contenido curado mediante hipervínculo dentro del texto?

Puntuación: 0-No; 1-Sí

- B3.2. Hipervínculo en apartado de lista de enlaces o etiquetas.

Pregunta de análisis: ¿El producto integra contenido curado mediante hipervínculo en un apartado específico de lista de enlaces o de etiquetas?

Puntuación: 0-No; 1-Sí

-B3.3. Contenido incrustado (embedded). 
Pregunta de análisis: ¿El producto integra contenido curado, incrustado o embebido manteniendo las funcionalidades de la plataforma original?

Puntuación: 0-No; 1-Sí

Los tres indicadores no son excluyentes y la puntuación del parámetro puede oscilar entre 1 y 3 .

\section{Ejemplos:}

- "Twitter y Facebook eliminan 359 cuentas falsas atribuidas al PP para las últimas generales", (Borja Andrino, Daniele Grasso, Jordi Pérez Colomé, El País, 21 septiembre 2019) <https://elpais.com/tecnologia/2019/09/20/actualidad/1568972332_038053. html>

\section{B3: 3 puntos}

Este artículo contiene los tres tipos de integración de contenido curado que se han mencionado: 1) existen hipervínculos dentro del texto que remiten a otros artículos, 2) hay asimismo hipervínculos en las etiquetas (tags) que acompañan al artículo, y por último, 3) se muestran publicaciones en Twitter incrustadas dentro del cuerpo del artículo. Se obtiene así 3 puntos correspondientes a los 3 indicadores del parámetro.

- "Fernando Alonso sufre en su estreno en Marruecos tras un triple pinchazo y pierde 45 minutos con Al-Attiyah" (20 Minutos, 5 octubre 2019) <https://www.20minutos.es/deportes/noticia/ fernando-alonso-sufre-marruecos-pierde-al-attiyah-3790354/0/>

\section{B3: 2 puntos}

El artículo inserta contenido procedente de Instagram junto a hipervínculos en el texto. Se obtiene así 2 puntos, que corresponden a los indicadores: hipervínculo dentro del texto, y contenido incrustado.

B4. Parámetro: Función periodística o informativa del contenido curado

\section{Definición:}

Este parámetro valora la función que cumple el contenido curado dentro del producto a analizar, desde el punto de vista periodístico o informativo.

Explicación:

Nos referimos en este apartado a la intencionalidad o propósito con el que cada contenido curado aparece dentro del producto periodístico analizado. A partir de Barnhurst (2013) y de Cui y Liu (2017), se pueden distinguir diferentes tratamientos o enfoques periodísticos en una curación periodística. De Cui y Liu se toman sus tres categorías principa- les, Sourcing curation, Contextualizing curation e Interpreting curation y se añaden cuatro más, obtenidas a partir de la observación de medios digitales realizada para esta investigación. Como en el parámetro anterior, la función se analiza exclusivamente para cada hipervínculo o contenido incrustado, y no en el resto de contenido redactado por el curador y que completa el producto en cuestión. Se consideran las siguientes categorías:

Sin modificar: contenido original sin modificar por parte del curador. Se han identificado dos tipos: hipervínculos en los que el texto corresponde al título original del documento, sin modificación por parte de este; y los documentos incrustados (embedded) procedentes de plataformas sociales.

Describir: Contenido curado como descripción o resumen. Coincide con sourcing curation: "Los hipertextos de los artículos reflejan parcial o totalmente el contenido de enlazado. Sin clicar el contenido original el lector debe ser capaz de tener una idea general del mismo" (Cui y Liu, 2017, 855). Este tratamiento periodístico está relacionado directamente con la técnica de sense making de resumir.

Contextualizar: el contenido curado se utiliza fundamentalmente para contextualizar o documentar una información (contextualizing curation): sin que ese contenido esté relacionado directamente con la historia de la noticia, puede ayudar sin embargo a entender los contextos sociales, culturales, históricos... de la misma. Se apoya especialmente en la curación de fuentes de información atemporal o retrospectiva.

Interpretar: el contenido curado es interpretado por el curador (interpreting curation), es decir, los hipervínculos a las fuentes no son frases descriptivas o de contexto sino interpretaciones o evaluaciones del contenido ("el contenido curado sirve para apoyar o reafirmar una opinión subjetiva o interpretativa del autor del texto", Cui y Liu, 2017, 859). Este tratamiento periodístico está relacionado directamente con la técnica de sense making de comentar.

Citar Fuente: cuando el texto del hipervínculo no se refiere a aquello de lo que trata el contenido al que se accede, sino que el hipervínculo señala cual es la fuente donde se ha publicado.

Citar Autor: como en el caso anterior, pero señalando al autor.

Llamar a la acción: la popular técnica del Call to Action (CtA) del marketing digital se utiliza también en los medios de comunicación digitales. Consiste en una llamada a entrar en un hipervínculo o en un contenido incrustado, a través de un texto del tipo "clica aquí". 


\section{Procedimiento:}

Para la evaluación de este parámetro se definen siete indicadores, uno para cada una de las categorías establecidas.

\section{- B4.1. Sin modificar}

Pregunta de análisis: ¿El producto analizado presenta acceso a contenido curado sin modificación en su título o directamente como contenido incrustado?

Puntuación: 0-No; 1-Sí

- B4.2. Describir.

Pregunta de análisis: ¿El producto analizado presenta acceso a contenido curado con un hipervínculo o incrustación de tipo descriptivo o de resumen?

Puntuación: 0-No; 1-Sí

\section{- B4.3. Contextualizar.}

Pregunta de análisis: ¿El producto analizado presenta acceso a contenido curado con un hipervínculo o incrustación del tipo de contextualización?

Puntuación: 0-No; 1-Sí

- B4.4. Interpretar.

Pregunta de análisis: ¿El producto analizado presenta acceso a contenido curado con un hipervínculo o incrustación de tipo interpretativo o de opinión?

Puntuación: 0-No; 1-Sí

- B4.5. Citar fuente.

Pregunta de análisis: ¿El producto analizado presenta acceso a contenido curado mediante indicación de la fuente?

Puntuación: 0-No; 1-Sí

- B4.6. Citar autor.

Pregunta de análisis: ¿El producto analizado presenta acceso a contenido curado mediante indicación del autor?

Puntuación: 0-No; 1-Sí

- B4.7. Llamar a la acción.

Pregunta de análisis: ¿El producto analizado presenta acceso a contenido curado mediante una llamada explícita a la acción?

Puntuación: 0-No; 1-Sí

Se trata de funciones no excluyentes y que se pueden complementar, aunque difícilmente se puede dar la combinación de todas ellas en un mismo producto; el mínimo de puntuación del parámetro es 1 y el máximo, 7 .

\section{Ejemplos:}

- "El Despertador, 9 de setembre: Deures per fer" (Ferran Cases, Nació Digital, 9 septiembre 2019)

<https://www.naciodigital.cat/noticia/186984/ deures/fer $>$

\section{B4: 5 puntos}

Los artículos y newsletters de este autor muestran una variada combinación de tratamientos periodísticos en sus contenidos curados. En este ejemplo se puede apreciar cómo: 1) describe, 2) contextualiza ("Així va explicar Macià..."), 3) interpreta ("tal com demostra..."), 4) cita autor ("article conjunt d'Oriol Junqueras i Marta Rovira) y 5) llama a la acción ("Recupereu-lo aquí"). Se obtiene así en este parámetro un total de 5 puntos correspondientes a los indicadores de: Describir, Contextualizar, Interpretar, Citar autor y Llamar a la acción.

- "Así hemos contado las primeras reacciones a la sentencia del 'procés' en Cataluña" (El Confidencial, 15 de octubre de 2019) https://www. elconfidencial.com/espana/cataluna/2019-10-15/ manifestaciones-el-prat-sentencia-proces-directo-cortes-vias-tren_2279696/"

\section{B4: 4 puntos}

En esta pieza periodística, del tipo de seguimiento en directo de un acontecimiento, se presenta el enlace al contenido curado de las siguientes maneras: 1) sin modificar (el más habitual en los contenidos curados procedentes de plataformas sociales, como tweets), 2) mediante hipervínculos descriptivos, 3) mediante citas al autor del contenido ("Lo cuenta Juanma Romero"), y 4) con llamadas a la acción ("Lea aquí la noticia completa"). Se obtienen así en este parámetro un total de 4 puntos correspondientes a los indicadores: Sin modificar, Describir, Citar autor, y Llamar a la acción.

\section{CONCLUSIONES}

El sistema que aquí se presenta es la primera propuesta existente en la literatura académica para el análisis y la evaluación de la curación de contenidos en periodismo. Hasta el momento se habían descrito casos, analizado muestras de diarios o propuesto taxonomías, pero no existía una propuesta de un sistema de análisis y evaluación, que se pueda aplicar a todo tipo de diarios o de productos de noticias, y que permita apreciar qué nivel de calidad existe en la curación de contenidos de un determinado diario o un determinado pro- 
ducto periodístico, ya sea un tipo de artículo, una sección, una newsletter... Este sistema de análisis va por tanto más allá de la simple descripción de una noticia o de un medio, pues permite, además de identificar sus componentes, establecer valoraciones de calidad, y realizar productos derivados, por ejemplo, ránquines, etc. En este sentido, nuestro sistema de análisis permite llevar a cabo al menos tres tareas distintas:

- realizar análisis comparativos entre diferentes productos noticiosos que utilicen la curación de contenidos;

- utilizar el sistema como herramienta para evaluar la calidad de la curación de contenidos por un medio concreto con el objetivo de identificar aquellos aspectos que son mejorables;

- utilizar el sistema como guía para el desarrollo de nuevos productos de calidad basados en la curación de contenidos.

En este sistema se ha potenciado la valoración de la variedad de usos de la curación, con el convencimiento de que una mayor variedad implica una mayor calidad de la misma. Así, por ejemplo, si observamos el parámetro A4, Fuentes del contenido curado atendiendo al tipo de organización, se considera que un producto periodístico que cure contenidos provenientes de varios tipos de fuentes informativas, tales como Organismos oficiales, Organizaciones privadas, Asociaciones, Ciudadanos y Medios de comunicación, tendrá una calidad superior a si solo ofrece contenidos de un solo tipo de fuentes de información, como por ejemplo Medios de comunicación (algo, esto último, muy habitual). En este caso, por tanto, cuanto mayor variedad de tipos de fuentes, mayor calidad en la curación. Así, en líneas generales consideramos aquí que la existencia de una mayor variedad de uso de cada uno de los elementos que conforman una curación, desde los tipos de fuentes a los tipos de técnicas de sense making, repercutirá en una mayor calidad de la curación y por extensión, en una mayor calidad del periodismo que se ofrece a la ciudadanía.

Como con cualquier sistema de análisis y evaluación, es importante señalar que éste debe es-

\section{REFERENCIAS}

Anton, L.; Guallar, J. (2014). Análisis de los archivos audiovisuales en internet de las televisiones autonómicas españolas. Revista Española de Documentación Científica, 37 (1), e033. https://doi.org/10.3989/ redc. 2014.1 .1044

Barnhurst, KG. (2013). Newspapers experiment online: Story content after a decade on the web. Journalism, v. 14, 3-21. https://doi.org/10.1177/1464884912448898 tar sujeto a pruebas y refinamientos sucesivos. Por nuestra parte, como futuras líneas de trabajo, en relación directa con este trabajo, se propone la aplicación de este sistema a muestras acotadas de medios digitales o de productos periodísticos; por ejemplo a: 1) medios digitales de un determinado país, principales medios de una área geográfica determinada o a nivel mundial; 2) productos periodísticos determinados, como newsletters, reportajes multimedia, redes sociales de medios...; - 3) contenidos informativos temáticos, como información política, deportiva, especializada en tecnología...

En suma, con esta propuesta y su aplicación sucesiva, se pretende ayudar a comprender mejor las potencialidades de la curación de contenidos en el periodismo, lo que puede revertir, tanto a nivel particular, en la mejora de su uso por parte de los medios digitales, como a nivel global, en la mejora de la calidad del periodismo, y tanto en escenarios profesionales como académicos y de investigación.

\section{AGRADECIMIENTOS}

Este estudio ha sido realizado en el marco del grupo de investigación consolidado "Cultura i Continguts Digitals" (SGR 2017-422), financiado por la Agència de Gestió d'Ajuts Universitaris i de Recerca (AGAUR) de la Generalitat de Catalunya y forma parte del proyecto "Narración interactiva y visibilidad digital en el documental interactivo y periodismo estructurado" RTI2018-095714-B-C21 (MICINN/FEDER), Ministerio de Ciencia, Innovación y Universidades (España).

\section{ACKNOLEDGEMENTS}

This study has been carried out within the framework of the consolidated research group "Cultura i Continguts Digitals" (SGR 2017-422), financed by the Agència de Gestió d'Ajuts Universitaris i de Recerca (AGAUR) of the Generalitat de Catalunya and is part of the project "Interactive narration and digital visibility in interactive documentary and structured journalism" RTI2018-095714-B-C21 (MICINN / FEDER), Ministry of Science, Innovation and Universities (Spain).

Bhargava, R. (2009). Manifesto For The Content Curator: The Next Big Social Media Job Of The Future?. Rohit Bhargava.com, 30 September. http://www. rohitbhargava.com/2009/09/manifesto-for-the-content-curator-the-next-big-social-media-job-of-thefuture-.html

Bhaskhar, M. (2016). Curation. The power of selection in a world of excess. [ebook] ISBN: 978-0349408699 
Bradshaw, P. (2013). Journalism *is* curation: tips on curation tools and techniques. Online journalism Blog, 30 September http://onlinejournalismblog. com/2013/09/30/curation-tools-tips-advice-journalism

Bruns, A. (2018). Gatewatching and news curation: Journalism, social media, and the public sphere. New York: Peter Lang. ISBN 9781433143984

Cappelletti Júnior, M.; Domínguez Quintas, S. (2014). La curaduría de contenidos y la narrativa colaborativa en el Ciberperiodismo: estudio del caso de Storify en el diario digital elpais.com. Estudios del mensaje periodístico, 20 (1) https://revistas.ucm.es/index.php/ ESMP/article/view/45216. https://doi.org/10.5209/ rev_ESMP.2014.v20.n1.45216

Carbonell Villena, N.; Pedraza-Jiménez, R. (2016). "Imágenes en prensa escrita". En: Pedraza-Jiménez, R.; Codina, L.; Guallar, J. Calidad en sitios web: método de análisis general, e-commerce, imágenes, hemerotecas y turismo, 93-128. Editorial UOC. Colección EPI Scholar.

Cascón-Katchadourian, J.; Ruiz-Rodríguez, A. A.; Alberich-Pascual, J. (2018). Revisión, análisis y evaluación de sistemas para la gestión de activos multimedia en organizaciones. Revista Española de Documentación Científica, 41 (1): e196. https://doi.org/10.3989/ redc. 2018.1 .1481

Codina, L. (2000). Evaluación de recursos digitales en línea. Revista Española de Documentación Científica, v. 23 , n. 1 , 9-44. https://doi.org/10.3989/redc. 2000. v23.i1.315

Codina, L. (2018). Curación de contenidos para periodistas: definición, esquema básico y recursos. Barcelona: Universitat Pompeu Fabra, Facultad de Comunicación, Área de Formatos Digitales e Interactivos, 32 p. https://repositori.upf.edu/handle/10230/34369

Creswell, J.W. (2009). Research Design. Qualitative, Quantitative and Mixed Methods Approach. London: Sage.

Cui, X.; Liu, Y. (2017). How does online news curate linked sources? A content analysis of three online news media. Journalism, 18 (7), 852-870. https://doi. org/10.1177/1464884916663621

Denzin, N. K.; Lincoln, Y. S. (2011). The Sage handbook of qualitative research. Thousand Oaks, California: Sage.

Deshpande, P. (2013). Six content curation templates for content annotation. The Curata blog, 13 agosto http:// www.curata.com/blog/6-content-curation-templates-for-content-annotation/

Díaz Arias, R. (2015). Curaduría periodística, una forma de reconstruir el espacio público. Estudios del mensaje periodístico, 21. http://revistas.ucm.es/index.php/ ESMP/article/view/51129

Díaz-Campo, J. (2014). Las cadenas de televisión españolas en Internet: un estudio sobre la calidad de sus sitios web. Estudios sobre el Mensaje Periodístico, 20 (1), 273-287. http://revistas.ucm.es/index.php/ ESMP/article/view/45219. https://doi.org/10.5209/ rev_ESMP.2014.v20.n1.45219

DIR Confidencial (2019). El ranking de audiencias de medios digitales, muy cerca de tocar techo tras estrecharse. DIR Confidencial, 23 enero. https://bit.ly/2BIrsie
Ferran-Ferrer, N.; Guallar, J.; Abadal, E.; Server, A. (2017). Research methods and techniques in Spanish library and information science journals (2012-2014). Information Research, 22 (1). http://eprints.rclis.org/32191/

Fondevila-Gascón, J F.; Segura-Jiménez, H. (2012). El peso de la hipertextualidad en el periodismo digital: estudio comparativo. Cuadernos de información, 30, 31-40. https://www.redalyc.org/pdf/971/97124309004.pdf

Glaser, B.; Strauss, A.L. (1967). The Discovery of Grounded Theory. Chicago: Aldine.

Guallar, J. (2017a). Artículos de curación de contenidos. Categorías y ejemplos. Anuario ThinkEPI, 11, 210-216 http://eprints.rclis.org/31800/. https://doi. org/10.3145/thinkepi.2017.38

Guallar, J. (2017b). Content curation in digital media: Between retrospective and real-time information. In: Campos Freire, F. et al. Media and Metamedia Management, Springer International Publishing, 37-46. ISBN: 9783319460666. https://doi.org/10.1007/978-3-31946068-0_6

Guallar, J.; Abadal, E. (2009). Evaluación de las hemerotecas de prensa digital: indicadores y ejemplos de buenas prácticas. El profesional de la información, 18 (3), 255-269. https://doi.org/10.3145/epi.2009.may.02

Guallar, J.; Abadal, E. ; Codina, L. (2012). Sistema de análisis de hemerotecas de prensa digital. Trípodos, 31, 37-64. http://raco.cat/index.php/Tripodos/article/view/262072

Guallar, J.; Codina, L. (2018). Journalistic content curation and news librarianship: Differential characteristics and necessary convergence. El profesional de la información, 27 (4), 778-791. https://doi.org/10.3145/ epi.2018.jul.07

Guallar, J.; Ferran-Ferrer, N.; Abadal, E.; Server, A. (2017). Revistas científicas españolas de información y documentación: análisis temático y metodológico. El profesional de la información, 26 (5), 947-960. https://doi.org/10.3145/epi.2017.sep.16

Guallar, J.; Leiva-Aguilera, J. (2013). El content curator. Guía básica para el nuevo profesional de internet. Barcelona: Editorial UOC, 2013. ISBN 978-84-9064-018-0

Guerrini, F. (2013). Newsroom curators \& independent storytellers: content curation as a new form of journalism. Reuters Institute for the Study of Journalism, University of Oxford. https://reutersinstitute.politics. ox.ac.uk/our-research/newsroom-curators-and-independent-storytellers-content-curation-new-form-journalism

Karlsson, M.; Clerwall, C.: Örnebring, H. (2015). "Hyperlinking practices in Swedish online news 2007-2013: The rise, fall, and stagnation of hyperlinking as a journalistic tool".. Information, communication \& society, 18 (7), 847-863. https://doi.or$\mathrm{g} / 10.1080 / 1369118 X .2014 .984743$

Linares, J.; Codina, L.; Abadal, E.; Guallar, J. (2016). Periodismo en Bases de Datos y Buscabilidad de la Información: protocolo de análisis. Hipertext.net: Anuario Académico Sobre Documentación Digital y Comunicación Interactiva, 14, 1-44. https://doi. org/10.2436/20.8050.01.33

Martínez-Cañadas, E. (2017). Curación de contenidos para bibliotecas. Barcelona: Editorial UOC, col. El profesional de la información, n. 42. ISBN: 9788490293706 
OJD Interactiva (2019). Medios digitales. https://www. ojdinteractiva.es/medios-digitales

Orero, P.; Cebrián-Enrique, B. J. (2019). Evolución del uso del hipertexto en la prensa española (2002-2016). El profesional de la información, 28 (2), e280225. https://doi.org/10.3145/epi.2019.mar.25

Parra-Valero, P. (2017). Curación de contenidos desde bibliotecas: competencias, herramientas y aplicaciones. Ciência da Informação, 45 (2), 103-117. http://revista.ibict.br/ciinf/article/view/3805

Pedraza-Jiménez, R.; Codina, L.; Guallar, J. (2016). Calidad en sitios web: método de análisis general, e-commerce, imágenes, hemerotecas y turismo. Barcelona: Editorial UOC. Colección EPI scholar.

Perpinyà Morera, R.; Cid-Leal, P. (2018). Los portales de archivos españoles: transparencia, interoperabilidad y orientación a los usuarios. Revista Española de Documentación Científica, 41 (3): e212. https://doi. org/10.3989/redc.2018.3.1507

Rodríguez-Martínez, R.; Codina, L.; Pedraza-Jiménez, R. (2010). Cibermedios y web 2.0: modelo de análisis y re- sultado de aplicación. El profesional de la información 19 (1), 35-44. https://doi.org/10.3145/epi.2010.ene.05

Rodríguez-Martínez, R.; Codina, L.; Pedraza-Jiménez, R. (2012). Indicadores para la evaluación de la calidad en cibermedios: análisis de la interacción y de la adopción de la Web 2.0. Revista Española de Documentación Científica, 35 (1), 61-93 https://doi.org/10.3989/ redc. 2012.1.858

Roig, N.; Pedraza-Jimenez, R. (2016). Comercio electrónico. En: Pedraza-Jimenez, R.; Codina, Ll.; Guallar, J. (coords.), Calidad en sitios web. Método de análisis general, e-commerce, imágenes, hemerotecas y turismo. Colección EPI scholar. Barcelona: Editorial UOC

Rosenbaum, S. (2011). Curation Nation: how to win in a world where consumers are creators. McGraw-Hil, 284 p. ISBN 978-0-07-176039-3

Sanabre, C.; Pedraza-Jiménez, R.; Codina, L. (2018). WebSite Canvas Model: propuesta de un modelo visual para la ideación estratégica de sitios web. Revista Española de Documentación Científica, 41 (4): e221. https://doi.org/10.3989/redc.2018.4.1542 